\title{
Investigation of Interface Orientation and Interface Mixing in Spin Valve Heterostructures
}

\author{
Hyun Sung Joo and Harry A. Atwater \\ Thomas J. Watson Laboratory of Applied Physics \\ California Institute of Technology, Pasadena, CA 91125
}

\begin{abstract}
We have investigated magnetoresistance properties of (100) epitaxial, (111) textured and polycrystalline spin valve heterostructures on (100) $\mathrm{Si}$ substrates by UHV ion beam sputtering at room temperature. Magnetoresistance was measured as a function of $\mathrm{Cu}$ interlayer thickness $\left(t_{\mathrm{i}}\right)$ with $10 \AA \leq t_{\mathrm{i}} \leq 100 \AA$ and the maximum was found at $20 \AA$ in the case of (100) epitaxial spin valves. Highly (111) textured spin valves with heterostructure configurations similar to $(100)$ spin valves were found to have a slightly lower magnetoresistance than (100) heterostructures, but a very similar functional dependence of magnetoresistance on $t_{\mathrm{i}}$.

Interface mixing during the sputtering process by energetic neutral bombardment was found to significantly affect the magnetoresistance. Samples were made under various sputtering conditions (gas pressure, ion beam energy, target and substrate configuration) that could enhance or suppress high energy neutral bombardment of the growing film surface. Samples made under the conditions that suppressed neutral bombardment showed higher magnetoresistance and more abrupt interfaces as confirmed by small angle X-ray diffraction (SAXD) analysis of interface mixing by energetic neutral bombardment during sputter deposition.
\end{abstract}

\section{INTRODUCTION}

Spin valve heterostructures of the form $\mathrm{Ni}_{80} \mathrm{Fe}_{20} / \mathrm{Cu} / \mathrm{Ni}_{80} \mathrm{Fe}_{20} / \mathrm{Fe}_{50} \mathrm{Mn}_{50}$ are of interest in the development of magnetoresistive magnetic reading heads of magnetic recording devices because of their soft magnetic properties and low saturation magnetic fields. [1] Spin valve effects, known as giant magnetoresistance, are associated with relative orientations of magnetization vectors in adjacent magnetic layers and they are very sensitive to crystallographic orientations and interface roughness.

Many investigators have reported magnetotransport properties related to crystalline texture and interface roughness in sputtered or $\mathrm{MBE}$ grown magnetic multilayers such as $\mathrm{Fe} / \mathrm{Cr}, \mathrm{Co} / \mathrm{Cr}, \mathrm{Co} / \mathrm{Cu}$ and $\mathrm{Fe} / \mathrm{Cu}$. [2]-[4] In investigations of spin valve heterostructures, the effects of (111) and random polycrystalline film texture [5] and interdiffusion induced by post-deposition annealing have been

Manuscript received February 17, 1995.

H. S. Joo, e-mail hjoo@daedalus.caltech.edu, phone 818-577-5754, fax 818-795-7258; H. A. Atwater, e-mail haa@daedalus.caltech.edu reported. [6] However, dependence of magnetoresistance on interface mixing and orientation of such multilayers was not clearly understood, but previous work indicated that magnetoresistance is sensitive to the method of film deposition. For an example MBE-grown $\mathrm{Co} / \mathrm{Cu}$ epitaxial films always showed lower magnetoresistance than sputtered polycrystalline samples, even though they had been expected to have unmixed sharp interfaces and well controlled crystalline orientations. [2]

In order to understand the effects of interface mixing and interface orientation in $\mathrm{NiFe} / \mathrm{Cu} / \mathrm{NiFe} / \mathrm{FeMn}$ spin valves, we have grown (100) epitaxial, (111) highly textured and polycrystalline spin valve structures by ultra high vacuum (UHV) ion beam sputtering with different seed layers, and measured their magnetotransport properties as a function of $\mathrm{Cu}$ interlayer thickness. In addition, energetic neutral bombardment was found to significantly affect the extent of interface mixing during the sputtering.

\section{EXPERIMENTAL DETÁILS}

Samples were prepared at room temperature by UHV ion beam sputtering at a base pressure lower than $2 \times 10^{-9}$ Torr. Samples discussed in this have the structures of $\mathrm{Si}(100) / \mathrm{seed}$ layer/50 $\AA \mathrm{Ni}_{80} \mathrm{Fe}_{20} d t_{\mathrm{i}} \AA \mathrm{Cu} / 50 \AA \mathrm{Ni}_{80} \mathrm{Fe}_{20} / 150 \AA \mathrm{Fe}_{50} \mathrm{Mn}_{50}$. $50 \AA(100)$ epitaxial $\mathrm{Cu}$ layers and $1000 \AA \mathrm{SiO}_{2} / 50 \AA \mathrm{Cu}$, $1000 \AA \mathrm{SiO}_{2}$ layers were used for (100) epitaxial and polycrystalline samples respectively. For (111) textured samples, seed layers were not used and multilayers were grown directly on (100) Si wafers. An in-plane $450 \mathrm{Oe}$ magnetic field was applied during growth by a permanent magnet. The deposition rate was $0.3-0.4 \AA / \mathrm{sec}$ and calibrated by Rutherford Back Scattering (RBS). Epitaxial (100) samples have been successfully grown on $50 \AA$ (100) epitaxial $\mathrm{Cu}$ seed layers. [7] Epitaxial (100) $\mathrm{Cu}$ seed layers were grown on hydrogen terminated Si (100) wafers. [8] Si wafers were cleaned by solution of $\mathrm{NH}_{4} \mathrm{OH}: \mathrm{H}_{2} \mathrm{O}_{2}: \mathrm{H}_{2} \mathrm{O}=$ 1:1:3 and dipped into 50:1 HF solution for 1 minute for hydrogen termination. (111) highly textured samples could be grown directly on hydrogen terminated (100) $\mathrm{Si}$ wafers. Polycrystalline samples were grown on $1000 \AA \mathrm{SiO}_{2}$ and 50 $\AA \mathrm{Cu} / 1000 \AA \mathrm{SiO}_{2}$.

The crystalline orientation of each layer was characterized by in situ Reflected High Energy Electron Diffraction (RHEED). X-ray diffraction was also used to confirm the textures of samples in large sample areas.

During the sputtering, Ar gas pressure, ion beam voltage 
and ion source-target-substrate angle were varied to enhance or to suppress interface mixing by energetic neutral bombardment. [20 $\AA \mathrm{Cu} / 20 \AA \mathrm{Pd}]_{15}$ multilayers deposited under identical sputtering conditions and seed layers to those of spin valve heterostructures were used for SAXD to survey the relative roughness of spin valve heterostructures. $\mathrm{Cu} / \mathrm{Pd}$ multilayers were chosen because Pd has a good X-ray contrast to $\mathrm{Cu}$ and it was reported that Pd (100) could be grown epitaxially on $\mathrm{Cu}(100)$. [9]

Resistance measurements were performed using a four point probe dc method. All resistance data in this article was measured at room temperature. A DC magnetic field was applied horizontally to the samples and parallel to the current, and varied from 100 to -100 Oe by means of a Helmholtz coil.

\section{RESULT AND DISCUSSION}

\section{A. Magnetoresistance and Interface Orientation}

Magnetoresistance (MR) was measured as a function of $\mathrm{Cu}$ interlayer thickness, $t_{\mathrm{i}}$, with $10 \AA \leq t_{\mathrm{i}} \leq 100 \AA$ and the maximum was found at $t_{\mathrm{i}}=20 \AA$ for both $(100)$ epitaxial and (111) textured films. For $t_{\mathrm{i}}<20 \AA$, MR decreased very quickly with thickness.(Fig. 1.). At $t_{\mathrm{i}}=10 \AA$, the spin valve effect vanished, possibly because of the ferromagnetic coupling of the free and pinned $\mathrm{NiFe}$ layers. Fig. 2. shows the interlayer exchange field $H_{\mathrm{i}}$ (defined as $H$ at $M R(H)=0.5$ $\left.M R_{\max }\right)$ as a function of the Cu interlayer thickness $t_{\mathrm{i}} \cdot H_{\mathrm{i}}$ drops very quickly below $20 \AA$ similar to MR..

The MR of highly (111) textured films with layer structures identical to (100) films was found to be slightly lower than the (100) films, but exhibited a very similar functional dependence on $t_{\mathrm{i}}$. The sample grown directly on $\mathrm{SiO}_{2}$ showed very small $\mathrm{MR}(\approx 0.1 \%)$ and spin valves grown on randomly oriented polycrystalline $\mathrm{Cu}$ seed layer structures identical to (100) epitaxial heterostructures did not show spin valve effects.

This result suggests that the crystallographic texture itself does not dominate spin valve effects in the $\mathrm{NiFe} / \mathrm{Cu} / \mathrm{NiFe} / \mathrm{FeMn}$ heterostructures, although it can affect exchange bias between the FeMn and the top NiFe film.

\section{B. Magnetoresistance and Interface Mixing}

In the ion beam sputtering process, controlling the neutral bombardment of the growing film surface by controlling process parameters such as ion beam voltage, Ar gas pressure and angle of ion source-target-sample is relatively easy in comparison to conventional sputtering or MBE. In spin valve magnetic multilayers, we found that interface mixing by energetic neutral bombardment of growing heterostructures during ion beam sputtering significantly affected the MR. Samples deposited with higher ion energies (higher ion beam voltage and lower argon pressure) had high resistance and

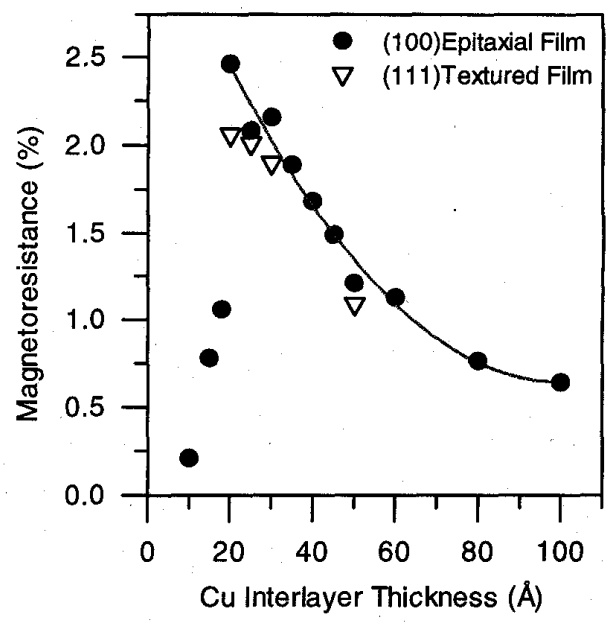

Fig. 1. Magnetoresistance as function of a Cu interlayer thickness, deposited at an ion beam voltage of $500 \mathrm{~V}, 8 \times 10^{-4}$ Torr Ar pressure for an ion sourcetarget-substrate angle that inhibits substrate irradiation by high energy neutral species.

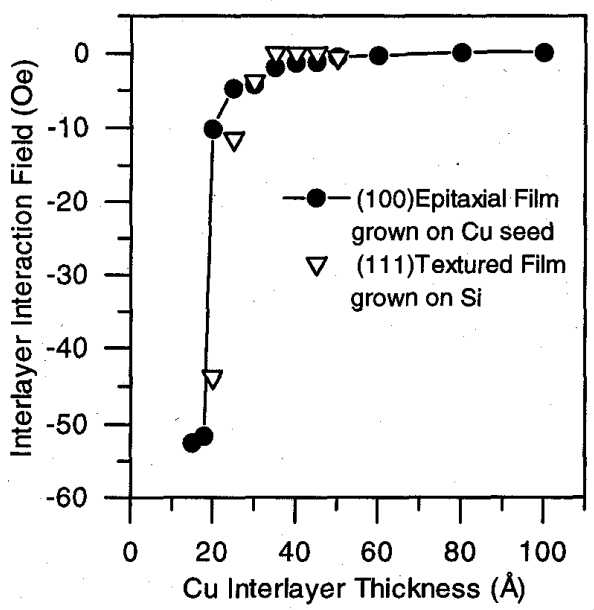

Fig. 2. Interface exchange fields as a function of $\mathrm{Cu}$ interlayer thickness, deposited at an ion beam voltage of $500 \mathrm{~V}, 8 \times 10^{-4}$ Torr Ar pressure for an ion source-target-substrate angle that inhibits substrate irradiation by high energy neutral species.

low MR. Samples deposited at $2 \times 10^{-4}$ Torr did not show spin valve phenomena. Fig. 3. illustrates the variation of $M R$ and resistance of samples deposited at various ion beam voltages. High energy neutral irradiation of growing film surfaces enhanced interface atomic mixing. The films grown with high energy neutral irradiation had high resistance and low MR due to interface mixing.

To minimize interface mixing by high energy bombardment, we located samples off-axis with respect to 
high energy neutrals from the sputtering target. With this configuration, the MR was dramatically increased. A doubling of the maximum MR from $1.2 \%$ to $2.45 \%$ was observed when the target was oriented so as to suppress bombardment of the growing heterostructure by high energy neutrals. To measure the change of interface mixing, SAXD experiments were carried out. We used $[20 \AA \mathrm{Cu} / 20 \AA \mathrm{Pd}]_{15}$ multilayer samples deposited under identical sputtering conditions as the $\mathrm{NiFe} / \mathrm{Cu} / \mathrm{NiFe} / \mathrm{FeMn}$ heterostructures. Multilayers of $\mathrm{Cu} / \mathrm{Pd}$ were used to avoid difficulties due to poor X-ray contrast of $\mathrm{NiFe}$ and $\mathrm{Cu}$. Diffraction data were

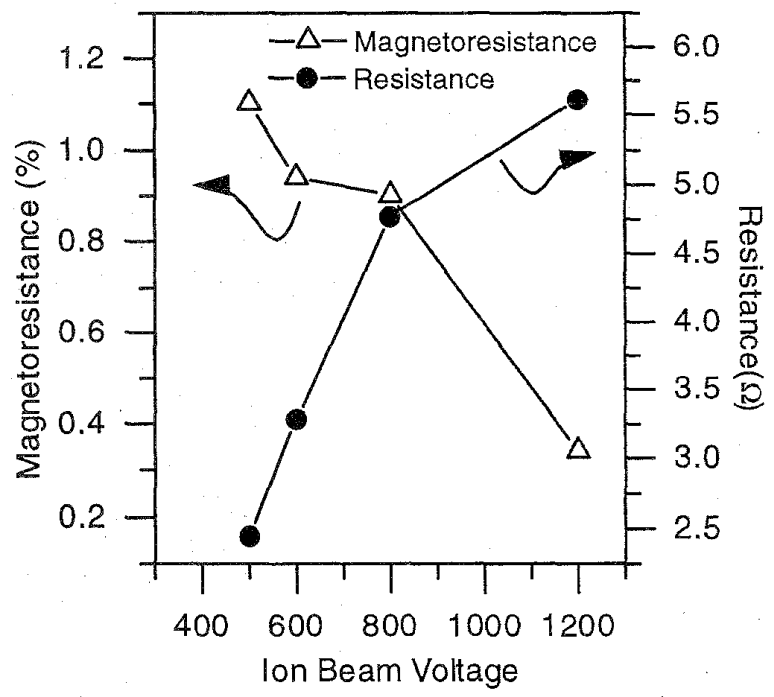

Fig. 3. Magnetoresistance and resistance of spin valves $\left(t_{\mathrm{i}}=20 \AA\right)$ deposited at different ion beam voltages, for an ion source-target-substrate angle that permits substrate irradiation by high energy neutral species.

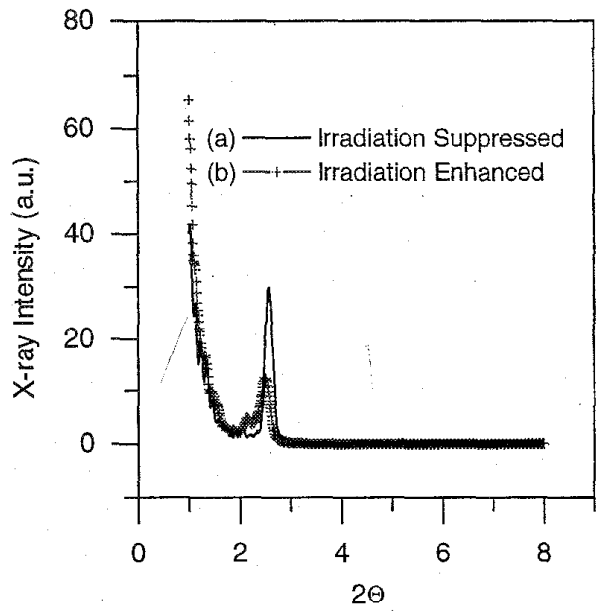

Fig. 4. Small angle $\mathrm{X}$-ray data of $[20 \AA \mathrm{Cu} / 20 \AA \mathrm{Pd}]_{15}$ multilayers deposited at an ion beam voltage of $500 \mathrm{~V}, 8 \times 10^{-4}$ Torr Ar pressure, for the substrate position that (a) suppressed irradiation and (b) enhanced irradiation consistent with MR data. We could obtain high intensity firstorder satellite peaks from the samples grown at off axis position(Fig. 4.), and they had high MR.

\section{CONCLUSION}

We found that interface mixing by energetic neutral bombardment during deposition significantly affected magnetotransport properties of $\mathrm{NiFe} / \mathrm{Cu} / \mathrm{NiFe} / \mathrm{FeMn}$ heterostructures whereas the interface crystallographic orientation alone did not significantly affect spin valve heterostructures. Spin valves with different texture showed similar functional trends with $\mathrm{Cu}$ interlayer thickness. Polycrystalline heterostructures without strong preferred orientations did not show spin valve behavior, possibly due to rough interfaces or small exchange bias fields.

\section{REFERENCES}

[1] B. Dieny, "Giant magnetoresistance in spin-valve multilayers", J. Magn. Magn. Mater., vol, 136, pp.335$359,1994$.

[2] W. F. Egelhoff and M. T. Kief, " $\mathrm{Fe} / \mathrm{Cu} / \mathrm{Fe}$ and $\mathrm{Co} / \mathrm{Cu} / \mathrm{Co}$ multilayers on $\mathrm{Cu}(111)$-The absence of oscillatory antiferromagnetic coupling", IEEE Trans. Mag., vol. 28, pp. 2742-2744, 1992.

[3] R. Q. Hood, L. M. Falicov, and D. R. Penn, "Effects of interfacial roughness on the magnetoresistance of magnetic metallic multilayers", Phys. Rev. B, vol. 49, pp. 368-377, 1994.

[4] K. I. Min. and S. K. Joo, "Enhancement of magnetoresistance sensitivity in a $\mathrm{Co} / \mathrm{Cu}$ artificial superlattice", J. Appl. Phys., vol. 75, pp. 4632-4635, 1994

[5] R. Nakatani, K. Hoshino, and Y. Sugita, "Magnetoresistance and preferred orientation in $\mathrm{Fe}$ $\mathrm{Mn} / \mathrm{Ni}-\mathrm{Fe} / \mathrm{Cu} / \mathrm{Ni}-\mathrm{Fe}$ sandwiches with various buffer layer materials", Jpn. J. Appl. Phys., vol. 33, pp. 133-137, 1994.

[6] V. S. Sperious, J. P. Nozieres, B. A. Gurney, B. Dieny, T. C. Huang, and H. Lefakis, "Role of interface mixing in giant magnetoresistance", Phys. Rev. B, vol. 47, pp. 11579-11582, 1993.

[7] I. Hashim, B. Park, and H. A. Atwater, "Epitaxial growth of $\mathrm{Cu}(001)$ on $\mathrm{Si}(001)$-Mechanism of orientation development and defect morphology", Appl. Phys. Lett., vol. 63 , pp. $2833-2835,1993$.

[8] I. Hashim and H. A. Atwater, "In-situ magnetic and structural analysis of epitaxial Ni80Fe20 thin films for spin valve heterostructures", $J$. Appl. Phys., vol. 75, pp. 6516-6518, 1994.

[9] C. A. Chang, "Deposition of (100) Au, Ag, Pd, Pt and Fe on (100) Si using different metal seed layers", J. Vac. Sci. Tech., vol. 9, pp. 98-101, 1991. 